\title{
DEVELOPMENT OF A METHOD OF ANALYSIS OF EEG WAVE PACKETS IN EARLY STAGES OF PARKINSON'S DISEASE
}

\author{
O.S. Sushkova ${ }^{1}$, A.A. Morozov ${ }^{1,2}$, A.V. Gabova ${ }^{3}$ \\ ${ }^{1}$ Kotel'nikov Institute of Radio Engineering and Electronics of RAS, Moscow, Russia \\ ${ }^{2}$ Moscow State University of Psychology \& Education, Moscow, Russia \\ ${ }^{3}$ Institute of Higher Nervous Activity and Neurophysiology of RAS, Moscow, Russia
}

\begin{abstract}
A method of analysis of EEG wave packets based on wavelets and nonparametric statistics is developed. The method is compared with standard methods based on Fourier spectra and complex Morlet wavelets by the example of Parkinson's disease experimental data. We demonstrate that these methods are complementary, that is, the standard methods and the wave packet analysis method reveal sufficiently different effects in the EEG data.
\end{abstract}

Keywords: wave packet, wave train, burst, electroencephalogram, EEG, wavelet, nonparametric statistics, decrease of quantity of wave packets, increase of alpha power spectral density.

Citation: Sushkova OS, Morozov AA, Gabova AV. Development of a method of analysis of EEG wave packets in early stages of Parkinson's disease. CEUR Workshop Proceedings, 2016; 1638: 681-690. DOI: 10.18287/1613-0073-20161638-681-690

\section{Introduction}

A wave packet is a wave action (a "burst") that is well localized in space and time. The wave packet is a typical pattern in a background electroencephalogram (EEG) and detecting / analysing such signals gives useful information about the brain activity. Alpha spindles (sleep spindles) and beta spindles are the best known examples of the wave packets in EEG; several methods based on Fourier spectra, wavelets, autoregressive models, adaptive filtering, etc. have been developed for detecting and analysing these EEG patterns (see surveys in $[1,2,3]$ ).

The idea of our method of EEG analysis is in that we detect and analyse the wave packets in a wide frequency band including theta, alpha, beta, and gamma EEG. We detect the wave packets as local maxima in a wavelet spectrogram of an EEG record and compute various attributes of these signals: a quantity of the wave packets per second, an average power / amplitude, an average central frequency, an average duration of the signals, etc. Then we implement a group statistical analysis on the base of 
these attributes using the nonparametric statistics. In the paper, we demonstrate that this method reveals a new effect in the early stages of Parkinson's disease.

The method of analysis of the EEG wave packets is described in Section "The Analysis Method". The experimental setting is described in Section "The Experimental Setting". The data processing stages and results of the statistical analysis are described in Section "Data Analysis". Section "Method Comparison" contains a comparison of the method with the standard methods based on Fourier spectra and complex Morlet wavelets. Section "Discussion" contains a discussion of the results of the statistical analysis and the comparison.

\section{The Analysis Method}

The method of EEG wave packet analysis includes detection of local maxima in wavelet spectrograms, determination of various attributes of these maxima, and statistical analysis of these attributes.

Let $M$ be a local maximum in a wavelet spectrogram. We consider $M$ as a case of a wave packet if the half width of $M$ (at half maximum) is greater or equal to the $T_{H}$ threshold in the time plane and greater or equal to the $F_{H}$ threshold in the frequency plane (see fig. 1). The $F_{H}$ threshold is a constant and the $T_{H}$ threshold is a function of the $f$ central frequency of the $M$ maximum:

$T_{H}=N_{P} /(2 f)$,

where $N_{\mathrm{P}}$ is a constant given by an expert. In this paper, we apply the values: $N_{P}=2$ and $F_{H}=1 \mathrm{~Hz}$.
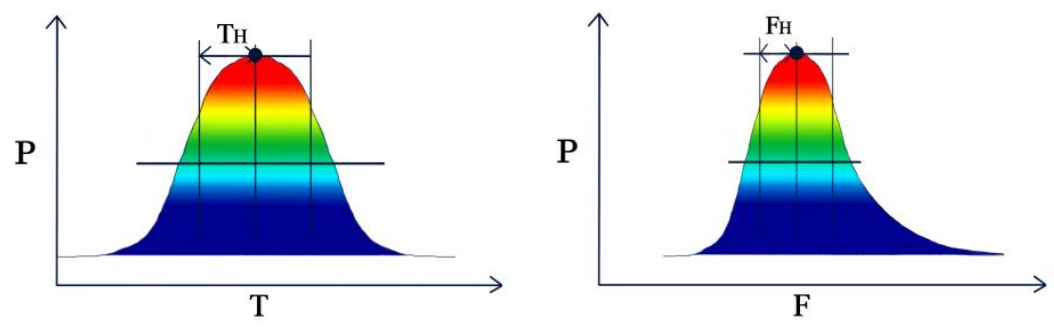

Fig. 1. An example of a spectrogram of a wave packet in a time-frequency domain. The diagram on the left shows the spectrogram of the signal in the time plane; the abscissa indicates a time and the ordinate indicates a power. The diagram on the right shows the spectrogram of the signal in the frequency plane; the abscissa indicates a frequency and the ordinate indicates a power

Let us consider a case of the wave packet analysis by the example of Parkinson's disease experimental data (fig. 2).

The amplitude of a typical wave packet in the alpha frequency band 8-12 Hz (an alpha spindle) is much bigger than the amplitude of a typical wave packet in the beta frequency band $12-25 \mathrm{~Hz}$ (a beta wave packet). Thus the beta wave packets are hardly noticeable against a background of the alpha wave packets. Moreover, spectrograms 
of alpha spindles have tails that continue in other frequency bands including the beta and the gamma bands. Thus classical EEG analysis methods based on wavelets can mistakenly account these tails of the alpha wave packets as a beta / gamma electrical activity.

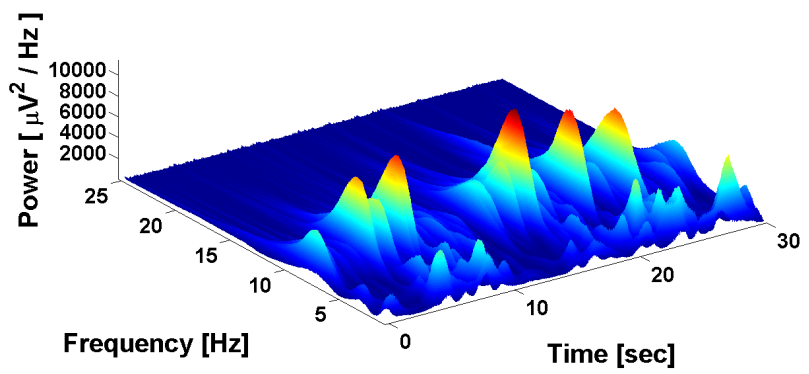

Fig. 2. A wavelet spectrogram of a background EEG record of an early stage Parkinson's disease patient. One can observe wave packets in the alpha frequency range 8-12 Hz well, but wave packets in the beta frequency range are hardly noticeable, because they are much smaller than the alpha wave packets

The method under consideration detects a set of wave packets in various frequency bands (fig. 3) in the complex Morlet spectrogram (fig. 4). Let us consider a spectrogram of a wave packet $B$ in a time-frequency domain (fig. 3 ) and a neighbourhood of the $B$ wave packet in the wavelet spectrogram (see fig. 5).

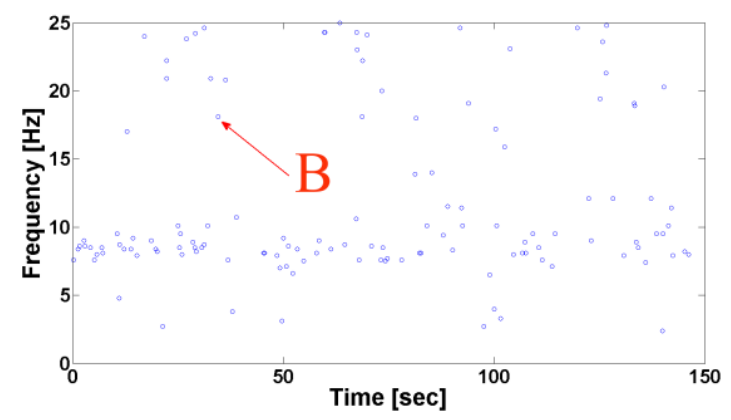

Fig. 3. A set of EEG wave packets in the time-frequency domain. The $B$ wave packet is indicated by an arrow

The $B$ wave packet has a distinct localization in the beta time-frequency area. At the same time, one can see a pronounced tail of another local maximum $A$ on the left that is originated in the alpha frequency band. The developed method prevents erroneous recognition of this tail as an electrical activity in the beta band. The original wave packet $B$ is shown in figure 6 . 


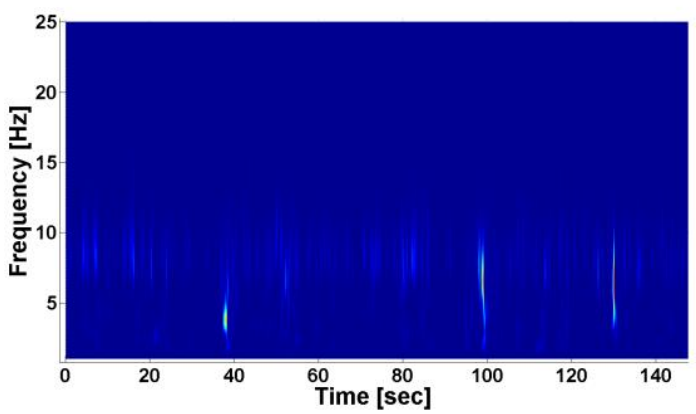

Fig. 4. An example of a wavelet spectrogram of an EEG record of an early stage Parkinson's disease patient (a view from above). The abscissa indicates a time (the $0-150 \mathrm{sec}$ interval) and the ordinate indicates a frequency (in $\mathrm{Hz})$

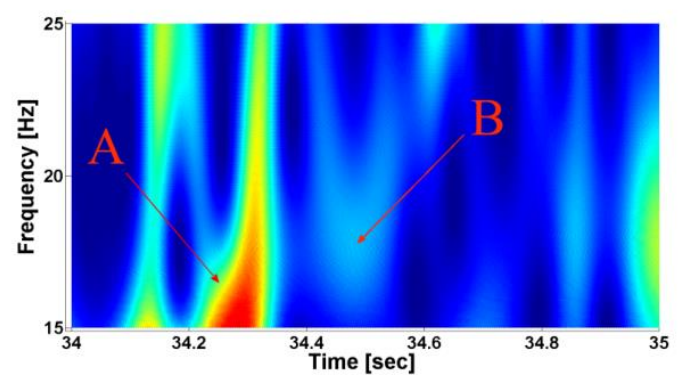

Fig. 5. A spectrogram of the $B$ wave packet in the beta band. One can see a pronounced tail of another local maximum $A$ on the left that is originated in the alpha band

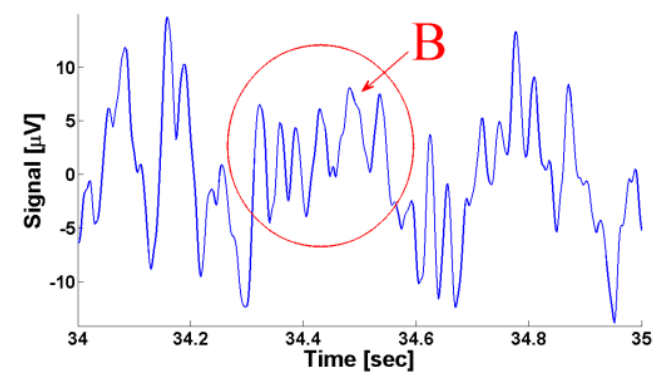

Fig. 6. The original EEG signal. The $B$ wave packet is indicated by an ellipse with an arrow

At the next stage of the analysis, a set of attributes of detected wave packets is calculated including the following ones:

- The quantity of the wave packets per second in given frequency bands.

- An average power / amplitude of the wave packets in the given bands.

- An average frequency of the wave packets in the given frequency bands. 
- A standard deviation of the power / amplitude of wave packets in the given frequency bands.

- An average duration of the wave packets in the given frequency bands.

Then the Mann-Whitney nonparametric statistical test is used for group comparisons of the calculated values. A nonparametric statistics is necessary because we analyze samples of small sizes (one can compare 15 subject groups and even less) and the samples do not correspond to the normal distribution law.

\section{The Experimental Setting}

A group of 18 patients with the first stage Parkinson's disease receiving no treatment and a group of 19 healthy volunteers as a control were recruited. The patients were diagnosed according to the standard Hoehn and Yahr scale. All patients and volunteers were right-handers. There were 11 patients with the right side tremor and $7 \mathrm{pa}$ tients with the left side tremor in the group. The age of patients ranged from 38 to 64 years; the mean age was 55 years; the standard deviation of the age was 6.5 years; the median of the age was 56 years; the $10 \%$ quantile of the age was 47.30 ; the $90 \%$ quantile was 62 years. The ages of controls ranged from 40 to 81 years; the mean age was 55 years; the standard deviation of the age was 10.51 years; the median was 53 years; the $10 \%$ quantile was 43.40 years; the $90 \%$ quantile was 72.60 years. No statistically significant differences between the ages of the patients and the controls were detected.

EEG was recorded in a non-standard condition [4], that is, a subject was instructed to keep a special pose to provoke a tremor: the arms were placed on armrests of the chair; the palms were straightened, placed in a vertical plane, and stretched a bit; the feet were stretched a bit and touched the floor by the heels only. The eyes were closed during the recording.

A 41-channel digital EEG system Neuron-Spectrum-5 (Neurosoft Ltd.) was used for the data acquisition. The sampling rate was $500 \mathrm{~Hz}$. The $0.5 \mathrm{~Hz}$ high-pass filter and the $50 \mathrm{~Hz}$ supply-line filter were used. Three EEG records were acquired for every subject with interruptions for a rest and a relaxation. The duration of every record was not less than 2 minutes. Then the best of three records was selected that contains a minimal number of artefacts.

A standard 10x20 EEG acquisition schema was used. In this paper, the C3 and C4 electrodes are considered only, because these electrodes approximately correspond to the motor cortex areas and are situated in the scalp area that produces a minimal number of muscle artefacts.

\section{Data Analysis}

Special software was developed for analyzing the data. The analysis includes the following EEG pre-processing operations: 
- The Huber's X84 method [5] for outlier rejection was used for removing EEG artefacts.

- A set of notch filters was applied for removing a power-line noise at 50, 100, 150, and $200 \mathrm{~Hz}$.

- The eight order 2-240 Hz band pass Butterworth filter was applied. Signals were filtered in the forward and reverse directions to eliminate a phase distortion.

- Signals were decimated with the decimation factor 8 .

The spectrograms were created using the Morlet wavelet:

$$
\Psi(x)=\frac{1}{\sqrt{\pi F_{b}}} \exp \left(2 i \pi F_{c} x\right) \exp \left(-\frac{x^{2}}{F_{b}}\right)
$$

In this paper, $F_{b}$ equals 1 and $F_{c}$ equals 1 . The frequency step in the spectrograms equals $0.1 \mathrm{~Hz}$.

The method of EEG wave packet analysis reveals a new effect in the Parkinson's disease, namely, the number of beta $(12-25 \mathrm{~Hz})$ wave packets in the $\mathrm{C} 3$ and $\mathrm{C} 4$ channels is significantly decreased (Mann-Whitney, $\mathrm{p}<0.02$ ), see figure 7 . Note that the quantity of the wave packets only is considered in this test, but not the amplitude of the wave packets.
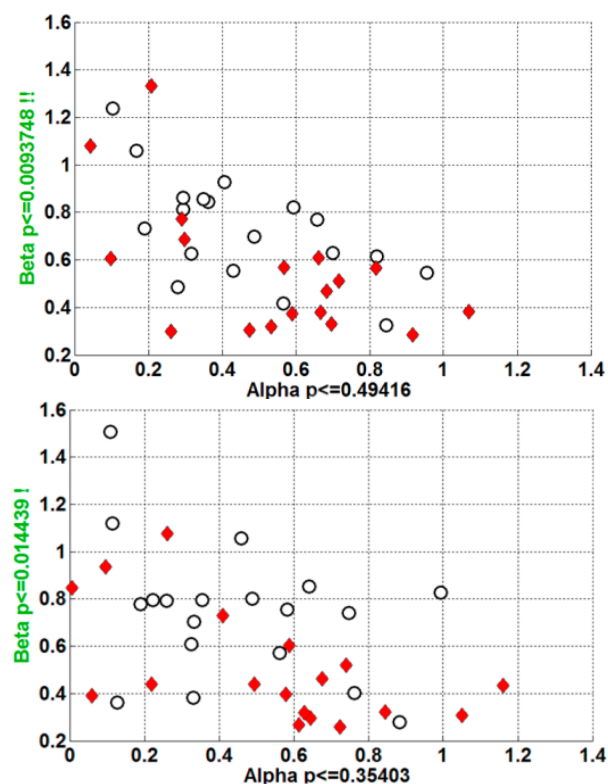

Fig. 7. Scattering of the quantity of beta wave packets in patients and controls. The $\mathrm{C} 3 \mathrm{elec}-$ trode is shown at the left, the $\mathrm{C} 4$ electrode is shown at the right. The abscissa is the number of wave packets per second in the alpha frequency band. The ordinate is the number of wave packets in the beta frequency band. The patients are indicated by diamonds and the controls are indicated by circles 


\section{Method Comparison}

Let us compare the developed method of EEG wave packet analysis with a standard method of EEG analysis based on the Welch spectra. Let us apply the following attributes of the Welch spectra:

- The signal is to be divided into 3 second segments.

- The Hann (Hanning) window is to be used.

- The overlap of the segments is $7 / 8$.

- The segment of the signal is to be padded with trailing zeros to smooth the spectra. The length of trailing zeros is $100 \%$ of the segment length.

Let us compute the Welch spectra of all patient and control EEG signals. Then compute mean values of each spectrum (a mean power spectral density) in the alpha $(8-12 \mathrm{~Hz})$ and beta $(12-25 \mathrm{~Hz})$ frequency bands. After that compare the patient and control samples of the mean power spectral density in given frequency bands.

The Mann-Whitney test indicates that there is a significant difference between the patient and control samples $(\mathrm{p}<0.05)$ in the alpha frequency band in the $\mathrm{C} 4$ electrode. In the $\mathrm{C} 3$ electrode, the test indicates only a statistical tendency in the alpha band (fig. 8).
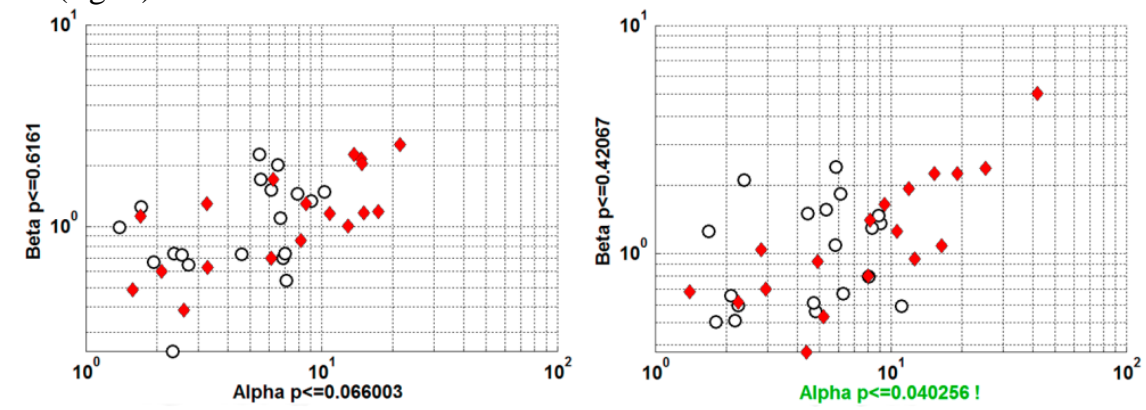

Fig. 8. Scattering of the mean power spectral density in patients and controls. The C3 electrode is shown at the left, the $\mathrm{C} 4$ electrode is shown at the right. The abscissa is a mean power spectral density in the alpha frequency band in a logarithmic scale. The ordinate is a mean power spectral density in the beta frequency band in a logarithmic scale. The patients are indicated by diamonds and the controls are indicated by circles

Thus the Welch spectra indicate the significant difference in Parkinson's disease in the alpha frequency band and no significant differences in the beta band. The developed method of EEG wave packet analysis and the standard Welch spectra are complimentary, that is, one can apply them simultaneously and get sufficiently different information about the subjects.

Let us compare the developed method with the standard one based on the complex Morlet wavelets. Firstly, compute wavelet spectrograms of all patient and control signals in the alpha and beta frequency bands with the $0.1 \mathrm{~Hz}$ frequency step. Then compute mean power spectral density of every spectrogram in every frequency band and apply the Mann-Whitney test to compare the patient and control samples. 
The Mann-Whitney test indicates a significant difference $(\mathrm{p}<0.03)$ between the patients and controls in the $\mathrm{C} 3$ and $\mathrm{C} 4$ electrodes in the alpha band (fig. 9).
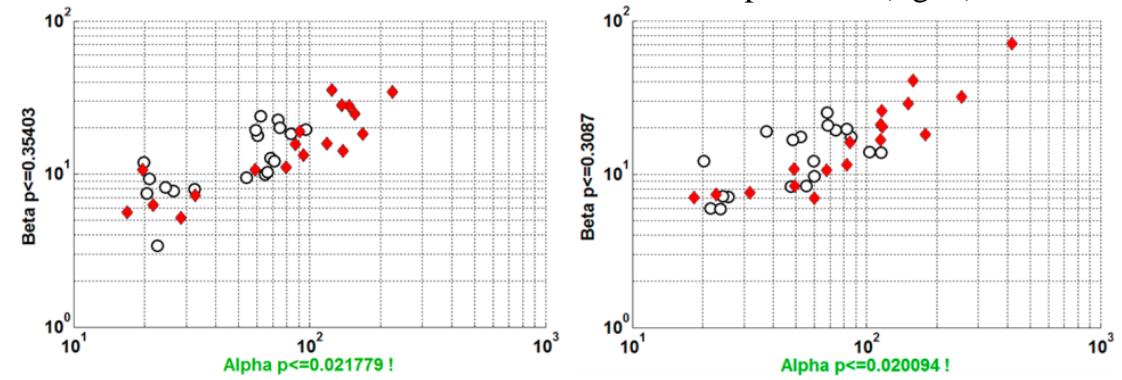

Fig. 9. Scattering of the wavelet mean power spectral density in patients and controls. The marking is the same as in the figure 8

Let us conduct another test. Compute medians of the power spectral density values of every spectrogram in every frequency band instead of averaging the values. The values of medians are shown in the figure 10.
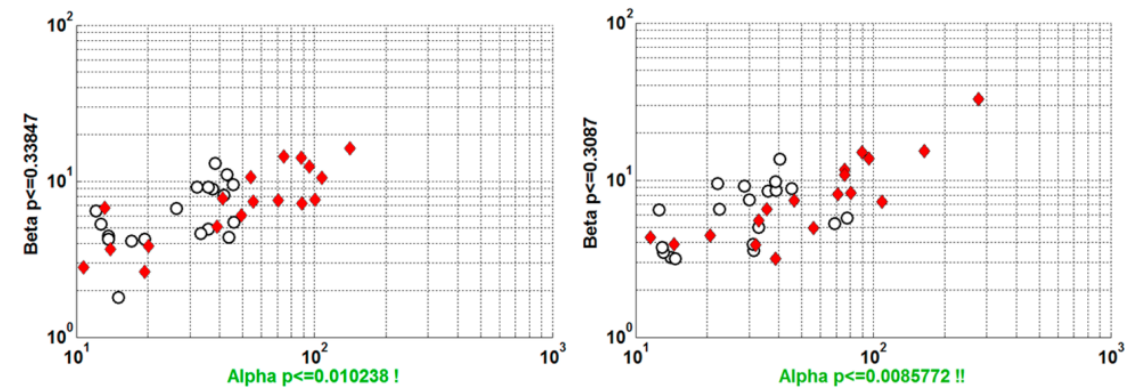

Fig. 10. Scattering of the medians of the wavelet power spectral density in patients and controls. The marking is the same as in the figure 8

The Mann-Whitney test confirms the significant differences in the $\mathrm{C} 3$ and $\mathrm{C} 4$ electrodes in the alpha band, but the probability of the first type error $(p<0.01)$ is better than in the foregoing comparisons with the Welch power spectral density and the wavelet mean power spectral density. Nevertheless, no significant differences are revealed in the beta frequency band.

\section{Discussion}

There are contradictory results reported in the neurophysiologic papers on the Parkinson's disease. For instance, in [6] a significant increase of the power spectral density in the beta frequency band in Parkinson's disease is reported. At the same time, a significant decrease of the beta power spectral density is reported in $[7,8]$. Our study shows that a detailed time-frequency dynamics of the cortex electrical activity in the alpha and beta frequency bands is to be considered to resolve the contradiction, be- 
cause a set of sufficiently different effects present in the neighbouring frequency bands, namely, a significant increase of the spectral density power in the alpha band and a significant decrease of the quantity of the wave packets in the beta frequency band. Furthermore, it is necessary to avoid an erroneous interpretation of the tails of the alpha wave packets as an electrical activity in the beta band.

The developed method of the analysis of EEG wave packets considers separate wave packets in the EEG signals and, therefore, analyzes the brain electrical activity more carefully. Nevertheless, the method is complementary with respect to the standard EEG analysis methods based on the power spectral density estimation, because these methods may discover different significant regularities in the EEG signals.

\section{Conclusion}

The method of a brain electrical activity investigation based on the EEG wave packet analysis is developed. The method reveals a new statistically significant effect in a group of de novo Parkinson's disease patients. The comparison of the method with the standard analysis methods based on the Welch spectra and complex Morlet wavelets shows that these methods are complimentary, that is, the standard methods indicate a significant increase of the power spectral density in the Parkinson's disease in the C3 and $\mathrm{C} 4$ electrodes in the alpha frequency band and the method of wave packet analysis indicates a significant decrease of the quantity of the wave packets in these electrodes in the nearby beta frequency range. This result demonstrates that an accurate EEG analysis based on the investigation of the time-frequency dynamics of the electrical activity is necessary to resolve the contradiction existed in the neurophysiologic literature.

\section{Acknowledgment}

Authors are grateful to Galina D. Kuznetsova for a help and comments on the preliminary versions of the paper, Yuriy V. Obukhov for a help in the statement of the problem, and Nikolay A. Kuznetsov for his critical notes on the statistical analysis method.

We acknowledge a partial financial support from the Russian Foundation for Basic Research, grants 16-37-00426, 15-07-07846.

\section{References}

1. Lawhern V, Kerick S, Robbins K. Detecting alpha spindle events in EEG time series using adaptive autoregressive models. BMC Neuroscience, 2013; 14: 101. URL: http://www.biomedcentral.com/1471-2202/14/101.

2. Parekh A, Selesnick IW, Rapoport DM, Ayappa I. Sleep spindle detection using timefrequency sparsity. In IEEE Signal Processing in Medicine and Biology Symposium. Philadelphia, PA: IEEE, Dec. 2014: 1-6. 
3. O'Reilly C, Nielsen T. Automatic sleep spindle detection: benchmarking with fine temporal resolution using open science tools. Frontiers in Human Neuroscience, 2015; 9: 353. DOI: 10.3389/fnhum.2015.00353.

4. Andreeva Y, Khutorskaya O. EMGs spectral analysis method for the objective diagnosis of different clinical forms of Parkinson's disease. J. Electromyography and Clinical Neurophysiology, 1996; 36(3): 187-192.

5. Hampel FR, Ronchetti EM, Rousseeuw PJ, Stahel WA. Robust Statistics. The Approach Based on Influence Functions. New York: John Wiley \& Sons, 1986.

6. Moazami-Goudarzi M, Sarnthein J, Michels L, Moukhtieva R, Jeanmonod D. Enhanced frontal low and high frequency power and synchronization in the resting EEG of parkinsonian patients. NeuroImage, 2008; 41: 985-997.

7. Pezard L, Jech R, Ruzicka E. Investigation of non-linear properties of multichannel EEG in the early stages of Parkinson's disease. Clinical Neurophysiology, 2001; 112: 38-45.

8. Stoffers D, Bosboom J, Deijen J, Wolters E, Berendse H, Stam C. Slowing of oscillatory brain activity is a stable characteristic of Parkinson's disease without dementia. Brain, 2007; 130: 1847-1860. 